\title{
Numerical Modelling of Magnetic Shielding by a Cylindrical Ferrofluid Layer
}

\author{
Olga Lavrova $^{a}$, Viktor Polevikov ${ }^{b}$ and Sergei Polevikov ${ }^{c}$ \\ ${ }^{a}$ Faculty of Mechanics and Mathematics, Belarusian State University \\ Independence Ave. 4, 220030 Minsk, Belarus \\ ${ }^{b}$ Faculty of Applied Mathematics and Informatics, Belarusian State University \\ Independence Ave. 4, 220030 Minsk, Belarus \\ ${ }^{c}$ OppenheimerFunds, Inc \\ 225 Liberty Street, 2WFC, 16 Floor, New York, NY 10281, USA \\ E-mail(corresp.): lavrovaolga@mail.ru \\ E-mail: polevikov@bsu.by \\ E-mail: spolevikov@ofiglobal.com
}

Received May 7, 2018; revised December 15, 2018; accepted December 27, 2018

\begin{abstract}
A coupled method of finite differences and boundary elements is applied to solve a nonlinear transmission problem of magnetostatics. The problem describes an interaction of a uniform magnetic field with a cylindrical ferrofluid layer. Ferrofluid magnetisations, based on expansions over the Langevin law, are considered to model ferrofluids with a different concentration of ferroparticles. The shielding effectiveness factor of the cylindrical thick-walled ferrofluid layer is calculated depending on intensities of the uniform magnetic field and on thickness of the ferrofluid layer.
\end{abstract}

Keywords: transmission magnetostatics problem, finite difference method, boundary element method, magnetic fluid, shielding.

AMS Subject Classification: $35 \mathrm{Q} 60 ; 65$ N06; 65 N38.

\section{Introduction}

Currently, one of the most important fields of scientific research is a study of electromagnetic properties of composite materials used in radio and electronic engineering. It requires development of modelling methods and computational techniques for processes of interaction between electromagnetic fields and objects of different geometries and material structures which are important for

Copyright (c) 2019 The Author(s). Published by VGTU Press

This is an Open Access article distributed under the terms of the Creative Commons Attribution License (http://creativecommons.org/licenses/by/4.0/), which permits unrestricted use, distribution, and reproduction in any medium, provided the original author and source are credited. 
further engineering of technical devices. A special challenge is studying materials with nonlinear physical properties $[8,11]$, in particular soft magnetic materials [28]. Computational approaches of handling equilibrium states of ferrofluid systems with different geometries and free surfaces - drop, layer, capillary have been developed in $[16,17,18,19,23]$ for uniform applied magnetic fields.

Recent results related to shells and membrane theories of composite materials in mechanics and biology are presented in [1]. In particular shells are applied for suppression of vibrations by means of magnetic fields, see e.g. [21,22] for thin sandwich-like shells containing magnetorheological layers. One of considered research directions is a study of transmission processes for electromagnetic fields through shields and shells, see e.g. [7]. Active research is performed on shielding problems of electromagnetic fields by solid magnetized thin-walled layers and films, see e.g. [2,10,12,13,30,31,32,33,34]. Current paper is devoted to studying shielding properties of cylindrical thick-walled ferrofluid layers protecting against uniform magnetic fields, where ferrofluids of various concentration of ferroparticles are considered. Numerical solutions of the problem have been obtained for a simpler mathematical model in [24], neglecting the effect of magnetodipole interparticle interactions in ferrofluids.

\section{Mathematical model for the magnetostatics problem}

Magnetic fluids (ferrofluids) are stable colloidal suspensions of ferromagnetic nanoparticles in a nonmagnetic carrier-liquid (such as water, kerosene, transformer oil, organic compounds and others). The ferroparticles of size $10 \mathrm{~nm}$ are in Brownian motion inside the carried-liquid. The fluid composition results in magnetic properties of magnetic fluids affected by magnetic fields $[3,4,6,28]$. The magnetic permeability of ferrofluids $\mu_{f}$ is defined by the magnetisation law $M(H)$

$$
\mu_{f}=\mu_{0} \mu, \quad \mu=\mu(H)=1+M(H) / H,
$$

where $\mu_{0}=4 \pi \cdot 10^{-7} \mathrm{H} / \mathrm{m}$ denotes the vacuum permeability. $H=|\boldsymbol{H}|$ is the intensity of the magnetic field. Different models of the ferrofluid magnetisation should be used to model fluids with different concentration of ferroparticles [14]. Namely, the following relations are suggested in [14] based on expansions over the Langevin law $M_{L}(H)=M_{s} L\left(H / H_{*}\right)$ within the framework of the modified mean-field theory,

$$
\begin{aligned}
& M_{(1)}(H)=M_{L}(H), \\
& M_{(2)}(H)=M_{L}\left(H+\frac{1}{3} M_{L}(H)\right), \\
& M_{(3)}(H)=M_{L}\left(H+\frac{1}{3} M_{L}(H)+\frac{1}{144} M_{L}(H) \frac{d M_{L}(H)}{d H}\right) .
\end{aligned}
$$

Here $M_{s}$ is saturation magnetisation of the fluid, $L(t)=\operatorname{coth}(t)-1 / t$ is the Langevin function, $H_{*}=k T /\left(\mu_{0} m\right), k=1.38 \cdot 10^{-23} \mathrm{~J} / \mathrm{K}$ is the Boltzmann constant, $T$ is the absolute temperature of the fluid, $m$ is magnetic moment of a ferropaticle. 
In contrast to $M_{(1)}(H)$, the magnetisation laws $M_{(2)}(H)$ and $M_{(3)}(H)$ take into account the magnetodipole interparticle interactions [14]. The magnetisation $M_{(1)}(H)$ describes diluted or weakly-concentrated ferrofluids (the magnetic phase concentration $\left.\phi_{m}<10 \%\right), M_{(2)}(H)$ describes moderatelyconcentrated ferrofluids $\left(\phi_{m} \sim 10-12 \%\right.$ ), and $M_{(3)}(H)$ describes dense or highly-concentrated ferrofluids $\left(\phi_{m} \sim 12-18 \%\right)$ [14]. It is assumed that the ferroparticles are uniformly distributed inside the ferrofluid.

Every magnetisation law is characterized by initial magnetic susceptibility $\chi_{(i)}=\lim _{H \rightarrow 0}\left(d M_{(i)} / d H\right), i=\overline{1,3}$ :

$$
\chi_{L}=\frac{M_{s}}{3 H_{*}}, \quad \chi_{(1)}=\chi_{L}, \quad \chi_{(2)}=\chi_{L}+\frac{1}{3} \chi_{L}^{2}, \quad \chi_{(3)}=\chi_{L}+\frac{1}{3} \chi_{L}^{2}+\frac{1}{144} \chi_{L}^{3} .
$$

$\chi_{(i)}$ is a dimensionless parameter of the ferrofluid. We formulate expressions for the relative magnetic permeability in terms of the dimensionless field intensity $h=H / H_{*}$ :

$$
\begin{aligned}
& \mu_{(1)}(H)=\bar{\mu}_{(1)}(h)=1+3 \chi_{L} \frac{L(h)}{h}, \\
& \mu_{(2)}(H)=\bar{\mu}_{(2)}(h)=1+3 \chi_{L} \frac{L\left(h+\chi_{L} L(h)\right)}{h}, \\
& \mu_{(3)}(H)=\bar{\mu}_{(3)}(h)=1+3 \chi_{L} \frac{L\left(h+\chi_{L} L(h)+\frac{1}{16} \chi_{L}^{2} L(h) \frac{d L(h)}{d h}\right)}{h} .
\end{aligned}
$$

\subsection{Differential statement of the problem}

Let us introduce a Cartesian coordinate system $O X Y Z$ and the corresponding cylindrical one $O \rho \varphi Z$ in $\mathbb{R}^{3}$. We consider a cylindrical layer, infinite in $Z$ direction and described by its cross-section

$$
D_{2}=\left\{(\rho, \varphi): R_{1}<\rho<R_{2}, 0 \leq \varphi<2 \pi\right\},
$$

with layer boundaries $\Gamma_{1}$ and $\Gamma_{2}$ such as

$$
\Gamma_{1}=\left\{(\rho, \varphi): \rho=R_{1}, 0 \leq \varphi<2 \pi\right\}, \quad \Gamma_{2}=\left\{(\rho, \varphi): \rho=R_{2}, 0 \leq \varphi<2 \pi\right\},
$$

see Figure 1.

The interfaces $\Gamma_{1}$ and $\Gamma_{2}$ are assumed to be absolutely permeable for magnetic fields. The inner domain $D_{1}=\left\{(\rho, \varphi): 0 \leq \rho<R_{1}, 0 \leq \varphi<2 \pi\right\}$ and the outer domain $D_{3}=\left\{(\rho, \varphi): R_{2}<\rho<\infty, 0 \leq \varphi<2 \pi\right\}$ are filled with vacuum of permeability $\mu_{0}$, whereas layer $D_{2}$ is filled with magnetic fluid of permeability $\mu_{f}$. The layer $D_{2}$ is considered under the effect of a uniform magnetic field $\boldsymbol{H}_{0}=H_{0} \boldsymbol{e}_{Y}$ with constant intensity $H_{0}$, where $\boldsymbol{e}_{Y}=(0,1,0)$, see Figure 1.

Let us denote $\boldsymbol{H}_{i}$ the magnetic field inside the domain $D_{i}$ and represent magnetic field of the external domain as $\boldsymbol{H}_{3}=\boldsymbol{H}_{0}+\tilde{\boldsymbol{H}}_{3}$. The applied field $\boldsymbol{H}_{0}$ does not depend on $Z$ coordinate. That is why the problem can be treated as two-dimensional in the polar coordinate system $O \rho \varphi$. Let us introduce 


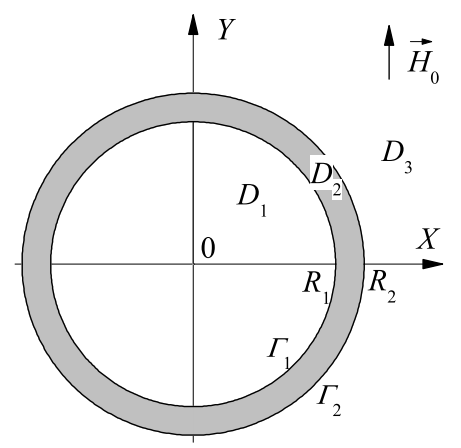

Figure 1. The problem setting: $D_{1}$ corresponds to the internal nonmagnetic medium, $D_{2}$ corresponds to the ferrofluid, and $D_{3}$ corresponds to the external nonmagnetic medium.

magnetostatic potential functions $U_{i}(\rho, \varphi)$ such that

$$
\boldsymbol{H}_{i}=\nabla U_{i}=\left(\frac{\partial U_{i}}{\partial \rho}, \frac{1}{\rho} \frac{\partial U_{i}}{\partial \varphi}\right), \quad i=\overline{0,3} .
$$

Now let us formulate the boundary value problem for unknown potentials $U_{1}, U_{2}$ and $\tilde{U}_{3}=U_{3}-U_{0}$, see e.g. [24], with the Laplace equations in the nonmagnatic domains $D_{1}$ and $D_{3}$ :

$$
\begin{aligned}
& \nabla^{2} U_{1}=\frac{1}{\rho} \frac{\partial}{\partial \rho}\left(\rho \frac{\partial U_{1}}{\partial \rho}\right)+\frac{1}{\rho^{2}} \frac{\partial^{2} U_{1}}{\partial \varphi^{2}}=0 \quad \text { in } D_{1} \\
& \nabla^{2} \tilde{U}_{3}=\frac{1}{\rho} \frac{\partial}{\partial \rho}\left(\rho \frac{\partial \tilde{U}_{3}}{\partial \rho}\right)+\frac{1}{\rho^{2}} \frac{\partial^{2} \tilde{U}_{3}}{\partial \varphi^{2}}=0 \quad \text { in } D_{3},
\end{aligned}
$$

and the nonlinear Laplace-type equation in the ferrofluid domain $D_{2}$ :

$$
\nabla \cdot\left(\mu \nabla U_{2}\right)=\frac{1}{\rho} \frac{\partial}{\partial \rho}\left(\mu \rho \frac{\partial U_{2}}{\partial \rho}\right)+\frac{1}{\rho^{2}} \frac{\partial}{\partial \varphi}\left(\mu \frac{\partial U_{2}}{\partial \varphi}\right)=0 \quad \text { in } D_{2},
$$

with transmission conditions on interfaces $\Gamma_{1}$ and $\Gamma_{2}$ :

$$
\begin{aligned}
& U_{1}=U_{2}, \quad \frac{\partial U_{1}}{\partial \rho}=\mu \frac{\partial U_{2}}{\partial \rho} \quad \text { on } \Gamma_{1}, \\
& U_{2}=U_{3}, \quad \mu \frac{\partial U_{2}}{\partial \rho}=\frac{\partial U_{0}}{\partial \rho}+\frac{\partial \tilde{U}_{3}}{\partial \rho} \quad \text { on } \Gamma_{2},
\end{aligned}
$$

and a radiation condition

$$
\lim _{\rho \rightarrow \infty} \tilde{U}_{3}=0 \text {. }
$$

Here the potential of the external applied field is given as $U_{0}=H_{0} \rho \sin \varphi$. The permeability of the ferrofluid $\mu=\mu\left(\left|\nabla U_{2}\right|\right)$ is defined by one of the relations (2.1)-(2.3). The Laplace equation (2.5) for the potential $\tilde{U}_{3}=U_{3}-U_{0}$ follows from the Laplace equation for $U_{3}$ and the fact that $\nabla^{2} U_{0}=0$. The mathematical model is described by the Laplace equations (2.4) and (2.5), the nonlinear Laplace-type equation (2.6), transmission conditions $(2.7),(2.8)$ and radiation condition (2.9). 


\subsection{Integral reformulation}

Let us now introduce dimensionless variables and parameters, where space variables are dimensionless over $R_{1}$, and the magnetic field is dimensionless over $H_{*}$ :

$$
\begin{aligned}
& r=\frac{\rho}{R_{1}}, \quad x=\frac{X}{R_{1}}, \quad y=\frac{Y}{R_{1}}, \quad u_{i}(r, \varphi)=\frac{U_{i}\left(R_{1} r, \varphi\right)}{R_{1} H_{*}}, \\
& h_{i}=\frac{H_{i}}{H_{*}}=\sqrt{\left(\frac{\partial u_{i}}{\partial r}\right)^{2}+\left(\frac{1}{r} \frac{\partial u_{i}}{\partial \varphi}\right)^{2}}, \quad u_{0}=h_{0} r \sin \varphi, \quad h_{0}=\frac{H_{0}}{H_{*}}, \quad \delta=\frac{R_{2}}{R_{1}} .
\end{aligned}
$$

The corresponding dimensionless geometry is

$$
\begin{aligned}
& \Omega_{1}=\{(r, \varphi): 0<r<1,0 \leq \varphi<2 \pi\}, \Omega_{2}=\{(r, \varphi): 1<r<\delta, 0 \leq \varphi<2 \pi\}, \\
& \Omega_{3}=\{(r, \varphi): \quad \delta<r<\infty, 0 \leq \varphi<2 \pi\}, \\
& \gamma_{1}=\{(r, \varphi): \quad r=1,0 \leq \varphi<2 \pi\}, \quad \gamma_{2}=\{(r, \varphi): \quad r=\delta, 0 \leq \varphi<2 \pi\} .
\end{aligned}
$$

The dimensionless analog of the Laplace equation (2.4) in the bounded domain $\Omega_{1}$ can be reformulated as a boundary integral equation on the interface $\gamma_{1}$, based on Green's representation formula, see e.g. [5]:

$$
\pi u_{1}\left(\xi_{0}\right)+\int_{\gamma_{1}}\left[u_{1}(\xi) q^{*}\left(\xi_{0}, \xi\right)-q_{1}(\xi) u^{*}\left(\xi_{0}, \xi\right)\right] d \gamma_{1}(\xi)=0, \quad \forall \xi_{0} \in \gamma_{1} .
$$

Here $\xi_{0}=\left(r_{0}, \varphi_{0}\right)$ is a fixed source point, $\xi=(r, \varphi)$ is integration variable and $q_{1}(\xi)=\partial u_{1} / \partial r$ is normal derivative of the potential function on $\gamma_{1}$. The fundamental solution $u^{*}\left(\xi_{0}, \xi\right)$ for the plane Laplace equation is of the following form, see e.g. [29]:

$$
u^{*}\left(\xi_{0}, \xi\right)=-\ln \left|\xi-\xi_{0}\right|, \quad q^{*}\left(\xi_{0}, \xi\right)=\frac{\partial u^{*}\left(\xi_{0}, \xi\right)}{\partial r}
$$

where $\left|\xi-\xi_{0}\right|=\left(\left(r \cos \varphi-r_{0} \cos \varphi_{0}\right)^{2}+\left(r \sin \varphi-r_{0} \sin \varphi_{0}\right)^{2}\right)^{1 / 2}$ is the distance between points $\xi$ and $\xi_{0}$. Similarly, the dimensionless form of the Laplace equation (2.5) in the unbounded domain $\Omega_{3}$ with the radiation condition (2.9) can be reformulated as a boundary integral equation on the interface $\gamma_{2}$ :

$$
\pi \tilde{u}_{3}\left(\xi_{0}\right)-\int_{\gamma_{2}}\left[\tilde{u}_{3}(\xi) q^{*}\left(\xi_{0}, \xi\right)-\tilde{q}_{3}(\xi) u^{*}\left(\xi_{0}, \xi\right)\right] d \gamma_{2}(\xi)=0, \quad \forall \xi_{0} \in \gamma_{2}
$$

where $\tilde{u}_{3}=u_{3}-u_{0}$, and $\tilde{q}_{3}(\xi)=\partial \tilde{u}_{3} / \partial r$ is normal derivative of the potential function $\tilde{u}_{3}$ on $\gamma_{2}$. For the problem under study,

$$
\begin{aligned}
& u^{*}\left(\xi_{0}, \xi\right)=-\ln \left|2 \sin \frac{\varphi-\varphi_{0}}{2}\right|, \quad q^{*}\left(\xi_{0}, \xi\right)=-\frac{1}{2}, \quad \forall \xi_{0}, \xi \in \gamma_{1}, \\
& u^{*}\left(\xi_{0}, \xi\right)=-\ln \left|2 \delta \sin \frac{\varphi-\varphi_{0}}{2}\right|, \quad q^{*}\left(\xi_{0}, \xi\right)=-\frac{1}{2 \delta}, \quad \forall \xi_{0}, \xi \in \gamma_{2} .
\end{aligned}
$$


As a result, an integration of the term $u_{1}(\xi) q^{*}\left(\xi_{0}, \xi\right)$ in the boundary integral equation $(2.10)$ and the term $\tilde{u}_{3}(\xi) q^{*}\left(\xi_{0}, \xi\right)$ in $(2.12)$ turns out to be zero due to the constant value of $q^{*}\left(\xi_{0}, \xi\right)$, see $(2.13),(2.14)$, and the symmetry of the potential functions

$$
\begin{aligned}
& \int_{\gamma_{1}} u_{1}(\xi) q^{*}\left(\xi_{0}, \xi\right) d \gamma_{1}(\xi)=-\frac{1}{2} \int_{\gamma_{1}} u_{1}(\xi) d \gamma_{1}(\xi)=0, \quad \forall \xi_{0} \in \gamma_{1} \\
& \int_{\gamma_{2}} \tilde{u}_{3}(\xi) q^{*}\left(\xi_{0}, \xi\right) d \gamma_{2}(\xi)=-\frac{1}{2 \delta} \int_{\gamma_{2}} \tilde{u}_{3}(\xi) d \gamma_{2}(\xi)=0, \quad \forall \xi_{0} \in \gamma_{2}
\end{aligned}
$$

The mathematical model (2.4)-(2.9) will be redefined by means of boundary integral equations (2.10) and (2.12), whereas the radiation condition (2.9) is exactly fulfilled due to integral formulation (2.12). A symmetry of the problem setting with respect to the coordinate axes (see Figure 1) allows us to restrict the dimensionless computational domain to the positive quadrant, see Figure 2. Hereafter,

$$
\begin{aligned}
& \Omega_{1}=\{(r, \varphi): 0<r<1,0<\varphi<\pi / 2\}, \Omega_{2}=\{(r, \varphi): 1<r<\delta, 0<\varphi<\pi / 2\}, \\
& \Omega_{3}=\{(r, \varphi): \delta<r<\infty, 0<\varphi<\pi / 2\}
\end{aligned}
$$

and the layer boundaries are

$$
\gamma_{1}=\{(r, \varphi): r=1,0<\varphi<\pi / 2\}, \quad \gamma_{2}=\{(r, \varphi): r=\delta, 0<\varphi<\pi / 2\} .
$$

We introduce additional notation for space variables on interfaces $\gamma_{1}$ and $\gamma_{2}$ to handle the symmetry in the integral equations:

$\xi_{1}^{[\sigma]}=(\sigma, \varphi), \xi_{2}^{[\sigma]}=(\sigma, \pi-\varphi), \xi_{3}^{[\sigma]}=(\sigma, \pi+\varphi), \xi_{4}^{[\sigma]}=(\sigma, 2 \pi-\varphi)$ for $\sigma \in\{1, \delta\}$

Due to symmetry of the potential functions and relations (2.15), we reformulate the boundary integral equations $(2.10),(2.12)$ as

$$
\begin{aligned}
& \int_{0}^{\pi / 2} q_{1}\left(\xi_{1}^{[1]}\right)\left[u^{*}\left(\xi_{0}, \xi_{1}^{[1]}\right)+u^{*}\left(\xi_{0}, \xi_{2}^{[1]}\right)-u^{*}\left(\xi_{0}, \xi_{3}^{[1]}\right)-u^{*}\left(\xi_{0}, \xi_{4}^{[1]}\right)\right] d \varphi \\
& =\pi u_{1}\left(\xi_{0}\right), \quad \forall \xi_{0} \in \gamma_{1}, \\
& \int_{0}^{\pi / 2} \tilde{q}_{3}\left(\xi_{1}^{[\delta]}\right)\left[u^{*}\left(\xi_{0}, \xi_{1}^{[\delta]}\right)+u^{*}\left(\xi_{0}, \xi_{2}^{[\delta]}\right)-u^{*}\left(\xi_{0}, \xi_{3}^{[\delta]}\right)-u^{*}\left(\xi_{0}, \xi_{4}^{[\delta]}\right)\right] d \varphi \\
& \quad=-\frac{\pi}{\delta} \tilde{u}_{3}\left(\xi_{0}\right), \quad \forall \xi_{0} \in \gamma_{2} .
\end{aligned}
$$

The mathematical model in dimensionless variables (see Figure 2) consists of boundary integral equations (2.16) and (2.17), together with the nonlinear Laplace-type equation

$$
\frac{1}{r} \frac{\partial}{\partial r}\left(\bar{\mu}\left(h_{2}\right) r \frac{\partial u_{2}}{\partial r}\right)+\frac{1}{r^{2}} \frac{\partial}{\partial \varphi}\left(\bar{\mu}\left(h_{2}\right) \frac{\partial u_{2}}{\partial \varphi}\right)=0 \quad \text { in } \Omega_{2},
$$




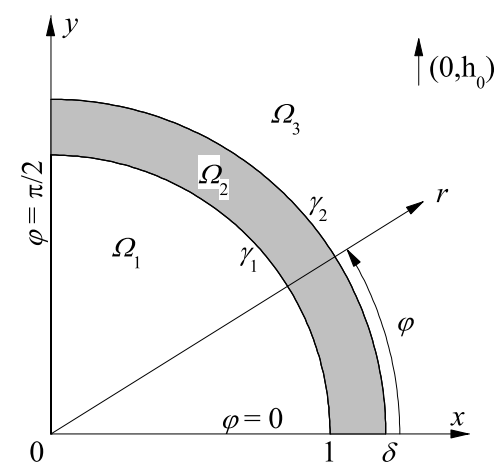

Figure 2. Computational domain for the problem under study.

as well as transmission conditions on interfaces $\gamma_{1}$ and $\gamma_{2}$ :

$$
\begin{aligned}
& u_{1}=u_{2}, \quad q_{1}=\bar{\mu}\left(h_{2}\right) q_{2} \quad \text { on } \gamma_{1}, \\
& u_{2}=\tilde{u}_{3}+h_{0} \delta \sin \varphi, \quad \bar{\mu}\left(h_{2}\right) q_{2}=\tilde{q}_{3}+h_{0} \sin \varphi \quad \text { on } \gamma_{2},
\end{aligned}
$$

and the symmetry conditions

$$
\begin{aligned}
& \left.u_{1}\right|_{\varphi=0}=\left.u_{2}\right|_{\varphi=0}=\left.\tilde{u}_{3}\right|_{\varphi=0}=0 \\
& \left.\frac{\partial u_{1}}{\partial \varphi}\right|_{\varphi=\frac{\pi}{2}}=\left.\frac{\partial u_{2}}{\partial \varphi}\right|_{\varphi=\frac{\pi}{2}}=\left.\frac{\partial \tilde{u}_{3}}{\partial \varphi}\right|_{\varphi=\frac{\pi}{2}}=0 .
\end{aligned}
$$

Here the normal derivatives of potential functions on $\gamma_{1}$ and $\gamma_{2}$ are denoted as $q_{1}=\partial u_{1} / \partial r, q_{2}=\partial u_{2} / \partial r$ and $\tilde{q}_{3}=\partial \tilde{u}_{3} / \partial r$. The unknown quantities of the mathematical model $(2.16)-(2.22)$ are the volume potential $u_{2}(r, \varphi)$ and the boundary fluxes $q_{1}(1, \varphi)$ and $\tilde{q}_{3}(\delta, \varphi)$. The mathematical model has three dimensionless parameters: applied field intensity $h_{0}$, layer thickness $\delta$ and magnetic susceptibility $\chi$.

Relative magnetic permeability of the ferrofluid $\bar{\mu}$ could take different forms such as $\bar{\mu}_{(i)}, i=\overline{1,3}$, see (2.1)-(2.3). The corresponding model formulations are denoted as models $i$, whereas the model 1 describes weakly-concentrated ferrofluids, the model 2 describes moderately-concentrated ones, and the model 3 corresponds to dense ferrofluids.

\section{Computational procedure}

The problem under study is not only of practical interest. It is also unique from the point of view of its numerical modelling. The computational algorithm for the integro-differential model (2.16)-(2.22) is based on the combination of the boundary element method in the nonmagnetic domains $\Omega_{1}$ and $\Omega_{3}$ and the finite-difference method in the ferrofluid domain $\Omega_{2}$. Due to the boundary element approach, a solution of the plane Laplace equation with known fundamental solution (see e.g. [29]) is replaced in the nonmagnetic domains with an 
equivalent problem of solving boundary integral equations (2.16) and (2.17). These equations are considered the Fredholm integral equations of the first kind with respect to the normal derivative $q_{1}$ on the interface $\gamma_{1}$ and to the normal derivative $\tilde{q}_{3}$ on the interface $\gamma_{2}$, respectively. An advantage of the boundary element method is that no meshes are required in the domains $\Omega_{1}$ and $\Omega_{3}$, and the radiation condition (2.9) is exactly fulfilled by the integral reformulation.

We apply a collocation approach with constant elements to approximate boundary integral equations (2.16) and (2.17) on uniform interface meshes $\left\{\left(1, \varphi_{j}\right)\right\}$ and $\left\{\left(\delta, \varphi_{j}\right)\right\}$ with respect to the integration variable $\varphi$, where $\varphi_{j}=$ $j \Delta \varphi, j=\overline{0, N_{\varphi}}$, and the mesh step $\Delta \varphi=\pi /\left(2 N_{\varphi}\right)$. For the given approximate values of the interface potentials $u_{j}^{(1)} \approx u_{1}\left(1, \varphi_{j}\right)$ and $\tilde{u}_{j}^{(3)} \approx \tilde{u}_{3}\left(\delta, \varphi_{j}\right)$, $j=\overline{1, N_{\varphi}}$, we get two independent systems of linear algebraic equations relative to the derivatives $q_{j}^{(1)} \approx q_{1}\left(1, \varphi_{j}\right)=\partial u_{1}\left(1, \varphi_{j}\right) / \partial r$ and $\tilde{q}_{j}^{(3)} \approx \tilde{q}_{3}\left(\delta, \varphi_{j}\right)=$ $\partial \tilde{u}_{3}\left(\delta, \varphi_{j}\right) / \partial r$ :

$$
\begin{aligned}
& \sum_{j=1}^{N_{\varphi}} a_{i j}^{[1]} q_{j}^{(1)}=\pi u_{i}^{(1)}, \quad i=\overline{1, N_{\varphi}} ; \quad u_{0}^{(1)}=q_{0}^{(1)}=0 \\
& \sum_{j=1}^{N_{\varphi}} a_{i j}^{[\delta]} \tilde{q}_{j}^{(3)}=-\frac{\pi}{\delta} \tilde{u}_{i}^{(3)}, \quad i=\overline{1, N_{\varphi}} ; \quad \tilde{u}_{0}^{(3)}=\tilde{q}_{0}^{(3)}=0
\end{aligned}
$$

where

$$
a_{i j}^{[\sigma]}=\int_{\varphi_{j-1 / 2}}^{\varphi_{j+1 / 2}}\left[u^{*}\left(\xi_{0}, \xi_{1}^{[\sigma]}\right)+u^{*}\left(\xi_{0}, \xi_{2}^{[\sigma]}\right)-u^{*}\left(\xi_{0}, \xi_{3}^{[\sigma]}\right)-u^{*}\left(\xi_{0}, \xi_{4}^{[\sigma]}\right)\right] d \varphi
$$

for $\sigma \in\{1, \delta\}$ and $\xi_{0}=\left(\sigma, \varphi_{i}\right)$. Due to a special form of the fundamental solution on $\gamma_{1}$ and $\gamma_{2}$, see $(2.16),(2.17)$, we get $a_{i j}^{[1]}=a_{i j}^{[\delta]}$. Hereafter we omit the superscript for $a_{i j}$ :

$$
a_{i j}=\int_{\varphi_{j-1 / 2}}^{\varphi_{j+1 / 2}} \ln \frac{\tan \left(\left(\varphi+\varphi_{i}\right) / 2\right)}{\tan \left(\left|\varphi-\varphi_{i}\right| / 2\right)} d \varphi \approx \Delta \varphi \ln \frac{\tan \left(\left(\varphi_{j}+\varphi_{i}\right) / 2\right)}{\tan \left(\left|\varphi_{j}-\varphi_{i}\right| / 2\right)}
$$

for $i=\overline{1, N_{\varphi}}, j=\overline{1, N_{\varphi}-1}, i \neq j$,

$$
\begin{aligned}
& a_{i i}=\int_{\varphi_{i-1 / 2}}^{\varphi_{i+1 / 2}} \ln \frac{\tan \left(\left(\varphi+\varphi_{i}\right) / 2\right)}{\tan \left(\left|\varphi-\varphi_{i}\right| / 2\right)} d \varphi \approx \Delta \varphi\left(1+\ln \frac{4 \tan \varphi_{i}}{\Delta \varphi}\right), i=\overline{1, N_{\varphi}-1}, \\
& a_{i, N_{\varphi}}=-\int_{\varphi_{N_{\varphi}-1 / 2}}^{\varphi_{N_{\varphi}+1 / 2}} \ln \tan \frac{\varphi-\varphi_{i}}{2} d \varphi \approx-\Delta \varphi \ln \tan \frac{\pi / 2-\varphi_{i}}{2}, i=\overline{1, N_{\varphi}-1} \\
& a_{N_{\varphi}, N_{\varphi}}=-\int_{\varphi_{N_{\varphi}-1 / 2}}^{\varphi_{N_{\varphi}+1 / 2}} \ln \tan \frac{|\pi / 2-\varphi|}{2} d \varphi \approx \Delta \varphi\left(1-\ln \frac{\Delta \varphi}{4}\right) .
\end{aligned}
$$

Note that singular integrals for the coefficients $a_{i i}$ are computed similarly to the approach in [5].

The nonlinear problem $(2.18),(2.21),(2.22)$ is approximated by the finitedifferences of the second order on a uniform mesh along $r$ and $\varphi$. The constructed finite-difference scheme is presented by a system of nonlinear algebraic 
equations. It satisfies the maximum principle in linear approximation at any mesh step, i.e. the finite-difference scheme is monotonic.

Computational process has been organized in the form of an iterative algorithm, where three mesh problems for the potential are solved independently of each other at each iteration.

1. At first, two linear BEM systems (3.1), (3.3) and (3.2), (3.3) are solved by the Gauss elimination method with respect to mesh values of $q^{(1)}$ and $\tilde{q}^{(3)}$, respectively, for the given mesh values of the potential $u^{(1)}$ and $\tilde{u}^{(3)}$ from the previous iteration. In spite of the fact that the problem is ill-posed in the theory of integral equations, we obtain only correct numerical solutions at different meshes.

2. Then, the interface mesh-values of $q^{(2)} \approx q_{2}$ on $\gamma_{1}$ at $r=1$ and on $\gamma_{2}$ at $r=\delta$ are computed from the transmission conditions (2.19) and (2.20) for the new mesh fluxes $q^{(1)}$ and $\tilde{q}^{(3)}$.

3. The nonlinear finite-difference problem with respect to the potential $u_{2}$ in the ferrofluid domain $\Omega_{2}$ is solved by the iterative Seidel-type method. As a result, new mesh values of the potential $u^{(2)} \approx u_{2}$ are determined not only at the internal nodes of the domain $\Omega_{2}$ but also at the interface nodes. New mesh values of the magnetic permeability $\bar{\mu}\left(h_{2}\right)$ are recomputed using these interface nodes.

4. At last, the mesh functions $u^{(1)}$ and $\tilde{u}^{(3)}$ are recomputed at the interface nodes, using transmission conditions (2.19) and (2.20). It completes the iteration of the computational process.

When the iterative process is finished, the potential $u_{1}(\xi)$ at any internal point $\xi \in \Omega_{1}$ and the potential $u_{3}(\xi)$ at any internal point $\xi \in \Omega_{3}$ are calculated by explicit formulas, based on the interface values of the potentials $u_{j}^{(1)}, u_{j}^{(3)}$ and the normal derivatives $q_{j}^{(1)}, \tilde{q}_{j}^{(3)}$ at the interface nodes $\left\{\left(1, \varphi_{j}\right)\right\},\left\{\left(\delta, \varphi_{j}\right)\right\}$ for $j=\overline{1, N_{\varphi}}$ :

$$
\begin{aligned}
& u_{1}\left(\xi_{0}\right)=\frac{1}{2 \pi} \sum_{j=1}^{N_{\varphi}}\left(a_{j}^{[1]} q_{j}^{(1)}-b_{j}^{[1]} u_{j}^{(1)}\right), \quad \forall \xi_{0} \in \Omega_{1}, \\
& u_{3}\left(\xi_{0}\right)=-\frac{\delta}{2 \pi} \sum_{j=1}^{N_{\varphi}}\left(a_{j}^{[\delta]} \tilde{q}_{j}^{(\delta)}-b_{j}^{[\delta]}\left(u_{j}^{(3)}-h_{0} \delta \sin \varphi_{j}\right)\right)+h_{0} r_{0} \sin \varphi_{0}, \quad \forall \xi_{0} \in \Omega_{3},
\end{aligned}
$$

where for $\sigma \in\{1, \delta\}$,

$$
\begin{aligned}
& a_{j}^{[\sigma]}=\Delta \varphi\left[u^{*}\left(\xi_{0}, \xi_{1, j}^{[\sigma]}\right)+u^{*}\left(\xi_{0}, \xi_{2, j}^{[\sigma]}\right)-u^{*}\left(\xi_{0}, \xi_{3, j}^{[\sigma]}\right)-u^{*}\left(\xi_{0}, \xi_{4, j}^{[\sigma]}\right)\right], \\
& b_{j}^{[\sigma]}=\Delta \varphi\left[q^{*}\left(\xi_{0}, \xi_{1, j}^{[\sigma]}\right)+q^{*}\left(\xi_{0}, \xi_{2, j}^{[\sigma]}\right)-q^{*}\left(\xi_{0}, \xi_{3, j}^{[\sigma]}\right)-q^{*}\left(\xi_{0}, \xi_{4, j}^{[\sigma]}\right)\right], \\
& \xi_{1, j}^{[\sigma]}=\left(\sigma, \varphi_{j}\right), \xi_{2, j}^{[\sigma]}=\left(\sigma, \pi-\varphi_{j}\right), \xi_{3, j}^{[\sigma]}=\left(\sigma, \pi+\varphi_{j}\right), \xi_{4, j}^{[\sigma]}=\left(\sigma, 2 \pi-\varphi_{j}\right) .
\end{aligned}
$$


The computations were carried out on a uniform mesh with the number of partitions $N_{r}=10$ for $\delta \leq 1.1$, and $N_{r}=100$ for $\delta>1.1$ for the variable $r \in[1, \delta]$, and $N_{\varphi}=100$ for the variable $\varphi \in[0, \pi / 2]$. The test calculations on a finer mesh with twice the number of partitions for both variables did not reveal any significant changes in the solution. An undisturbed magnetic potential $u_{2}=u_{0}$ was chosen as starting configuration for the iterative process.

\section{Results of computations}

The initial magnetic susceptibility $\chi$ lies in the range $0<\chi \leq 1$ for the weaklyconcentrated ferrofluids, and in the range $1<\chi \leq 10$ for the moderatelyconcentrated ones. It reaches values $\chi \sim 50-80$ for the dense ferrofluids under the room-temperature conditions $[20,26,27]$. For instance, the dense decanebased ferrofluid with magnetic phase $\phi_{m}=22.6 \%$ has initial susceptibility $\chi=51$ under the room temperature, see e.g. [9]. Under low temperatures ( $T \sim 230-240 \mathrm{~K})$, the value of the initial magnetic susceptibility of dense laboratory synthesized ferrofluids increases up to $\chi \sim 100-120$ for the preserved fluidity, see e.g. $[20,26,27]$. We take the initial magnetic susceptibility from the range $10 \leq \chi \leq 50$ for the test computations, namely $\chi=\{10,30,50\}$. The value $\chi=10$ corresponds to moderately-concentrated ferrofluids, whereas $\chi=\{30,50\}$ describes dense ferrofluids.

Shielding effectiveness factor of the uniform magnetic field $\boldsymbol{H}_{0}$ by the cylindrical thick-walled ferrofluid layer $D_{2}$ is defined by the formula

$$
K_{\mathrm{ef}}=\frac{\left|\boldsymbol{H}_{0}(0, \varphi)\right|}{\left|\boldsymbol{H}_{1}(0, \varphi)\right|}=\frac{h_{0}}{\left|\nabla u_{1}(0, \varphi)\right|}=h_{0} /\left.\sqrt{\left(\frac{\partial u_{1}}{\partial r}\right)^{2}+\left(\frac{1}{r} \frac{\partial u_{1}}{\partial \varphi}\right)^{2}}\right|_{r=0} .
$$

Shielding effectiveness factor $K_{\text {ef }}$ estimates the decay factor of the externally applied magnetic field intensity $H_{0}$ transmitted into the internal cylinder $D_{1}$. We compute (4.1) at a point where $\varphi=\pi / 2$, lying on the axis $O y$, with symmetry condition (2.22), hence

$$
K_{\mathrm{ef}}=h_{0} /\left|\lim _{r \rightarrow 0} \frac{\partial u_{1}(r, \pi / 2)}{\partial r}\right| .
$$

Figure 3 demonstrates the shielding effectiveness factor $K_{\text {ef }}$ dependent on the applied field for models 1-3. The geometric configuration of the problem is specified by the layer thickness $\delta=1.1$. The ferrofluid properties are defined two ways: by the initial magnetic susceptibility $\chi=\{10,30,50\}$ with $\chi_{(i)}=\chi$, see Figure 3a, and by the Langevin susceptibility $\chi_{L}=\{4.06,7.61,9.97\}$ with $\chi_{(i)}=\chi_{(i)}\left(\chi_{L}\right)$, see Figure $3 \mathrm{~b}$. The values of the susceptibilities are taken in such a way that $\chi_{(3)}\left(\chi_{L}\right)=\chi$, it leads to the identical numerical results for model 3 (solid lines) in Figure 3a and Figure 3b. Figure 3a illustrates the comparison of models 1-3, aligned for weak magnetic fields by the same values of $\chi$, whereas Figure 3b shows the comparison of models 1-3 with analogous physical ferrofluid properties, fixed by the same values of $\chi_{L}$. Numerical results in Figure 3 suggest that in weak fields $\left(h_{0}<0.1, H_{0}<10^{2} \mathrm{~A} / \mathrm{m}\right)$ the shielding factor preserves a constant maximum value, and the shielding effect is higher 
for more concentrated ferrofluids. Namely, $K_{\mathrm{ef}} \approx 1.4$ for the moderatelyconcentrated ferrofluid with $\chi=10$ and $K_{\mathrm{ef}} \approx 3.1$ for the dense ferrofluid with $\chi=50$, see Figure $3 \mathrm{a}$. We note that the numerical results of model $1-3$ for the same $\chi$, shown in Figure $3 \mathrm{a}$, coincide in weak magnetic fields due to the identical linear behavior of magnetisation laws $M_{(i)}=\chi_{(i)} H \rightarrow \chi H$ in the limit case $H \rightarrow 0$. As magnetic fields increase, the shielding effectiveness factor $K_{\text {ef }}$ monotonically decreases to 1 and the shielding effect is almost absent for $h_{0}>10^{2}\left(H_{0}>10^{6} \mathrm{~A} / \mathrm{m}\right)$. We conclude that the ferrofluid shield is effective in weak magnetic fields, whereas the concentration of magnetic particles could be considered as a way to control the intensity of the externally applied magnetic field, penetrating to the inner domain of the shield. For instance, geomagnetic storms on the Earth surface have intensity $H_{0}<200 \mathrm{~A} / \mathrm{m}$ and could be effectively shielded by ferrofluid layers. We note that numerical results in Figure 3a for the model 1 have been originally published in [24].

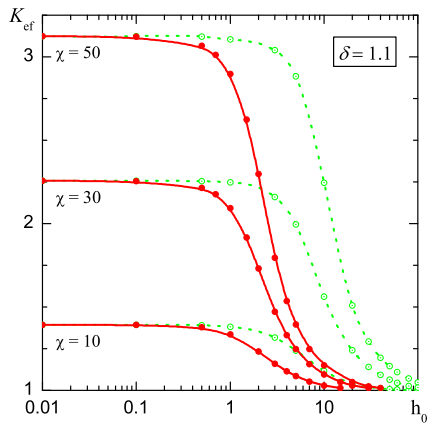

a)

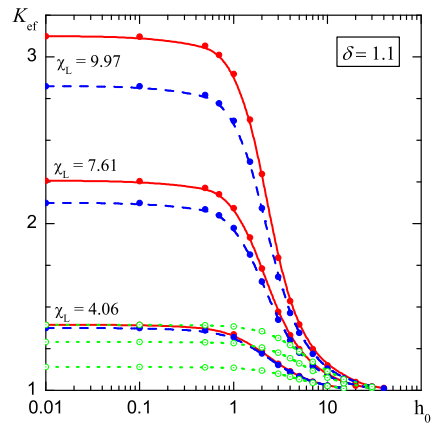

b)

Figure 3. Dependence of the shielding effectiveness factor $K_{\text {ef }}$ on the dimensionless applied magnetic-field intensity $h_{0}$ for the dimensionless layer thickness $\delta=1.1$. The ferrofluid properties are defined two ways: by the initial magnetic susceptibility $\chi=\{10,30,50\}$ (a) and by the Langevin permeability $\chi_{L}=\{4.06,7.61,9.97\}$ (b). Dotted lines correspond to model 1 , dashed lines to model 2, solid lines to model 3 . On the scale of the graph, the results for model 2 are indistinguishable from those of model 3 in (a). Lines for model 1 in (b) correspond to increasing values of $\chi_{L}$ bottom-up.

Figure $3 \mathrm{~b}$ allows to compare numerical results with respect to models $1-3$, where the ferrofluid properties are specified by the model-independent parameter $\chi_{L}$, in contrast to the parameter $\chi$ in Figure 3a. Figure 3b shows that different models produce quantitatively different results for the same ferrofluids. Namely, model 1 does not suit for describing moderately- and stronglyconcentrated ferrofluids $\left(\chi_{L}>1\right)$ for the problem under study. The shielding effect is underestimated for $\chi_{L}>1$ in the frame of model 1 . The results of model 2 and model 3 are quantitatively comparable for moderatelyconcentrated ferrofluids $\left(\chi_{L}=4.06\right)$, but the shielding effect is underestimated for strongly-concentrated ferrofluids $\left(\chi_{L}=7.61\right.$ and $\left.\chi_{L}=9.97\right)$ in the frame of model 2. We note that the numerical results of model 1-3 for the same $\chi_{L}$, shown in Figure 3b, coincide for magnetic fields $h_{0}>10$ due to the same behavior of magnetisation laws in saturation $M_{(i)}=\chi_{(i)} H \rightarrow M_{s}$ in the limit 
case $H \rightarrow \infty$.

The shielding effectiveness factor has an explicit analytical representation in a weak field limit, see e.g. [15, equation (72)] or [7, equation (B.34)]

$$
K_{\mathrm{ef}}=\frac{1}{4 \mu_{(0)}}\left(\left(\mu_{(0)}+1\right)^{2}-\frac{1}{\delta^{2}}\left(\mu_{(0)}-1\right)^{2}\right) .
$$

This formula corresponds to a linear magnetisation law of the ferrofluid $M_{(0)}(H)=\chi_{L} H$ with the initial susceptibility $\chi_{(0)}=\chi_{L}$ and the relative magnetic permeability $\mu_{(0)}=1+\chi_{L}$. To verify the computations, we compare the numerical results from Figure $3 \mathrm{a}$ for $h_{0}=0.01$ and $\chi=\{10,30,50\}$ with the analytical ones for $\chi=\chi_{L}, \delta=1.1$ and observe the agreement between them up to the fourth significant digit.

Figure 4 shows the shielding effectiveness factor $K_{\text {ef }}$ dependent on the layer thickness at the applied field $h_{0}=1$ for different values of the Langevin susceptibility $\chi_{L}=\{4.06,7.61,9.97\}$ and for models $2-3$. The layer thickness is presented here by the difference between the outer and the inner layer radii as $\delta-1=\left(R_{2}-R_{1}\right) / R_{1}$ and specified by the values $\delta-1 \in(0.01,10)$.

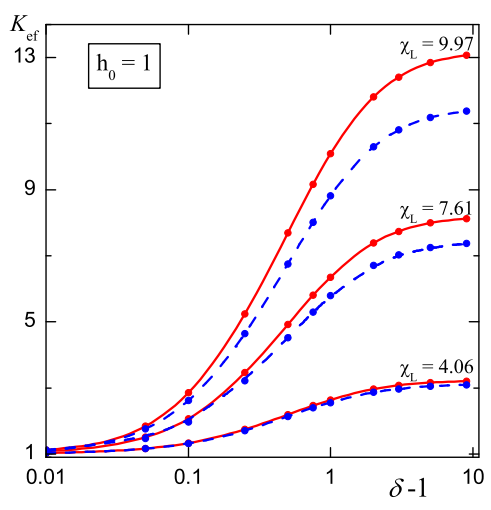

Figure 4. Dependence of the shielding effectiveness factor $K_{\mathrm{ef}}$ on the dimensionless layer thickness $\delta-1=\left(R_{2}-R_{1}\right) / R_{1}$ for the dimensionless applied magnetic-field intensity $h_{0}=1$ and the Langevin susceptibility $\chi_{L}=\{4.06,7.61,9.97\}$. Dashed lines correspond to model 2. Solid lines correspond to model 3.

Numerical results demonstrate the monotone increase of the shielding effect as the layer thickness increases. The strongest growth of shielding effectiveness factor is shown for $\delta-1 \in(0.01,3)$ with tendency to reach a constant value of $K_{\text {ef }}$ for thick layers with $\delta-1>5$. This qualitative behavior is present for ferrofluids of different concentrations, namely, for the moderately-concentrated ferrofluid with $\chi_{L}=4.06$ and for the highly-concentrated ferrofluids with $\chi_{L}=7.61$ and $\chi_{L}=9.97$. A stronger shielding effect is achieved for ferrofluids with a larger value of $\chi_{L}$ or a higher ferroparticle concentration, similarly to the observation in Figure 3. The shielding effectiveness factor is higher for model 3 than for model 2 at different layer thicknesses, whereas the difference between numerical results of models 2 and 3 increases with increasing the ferroparticle concentration. Results in Figure 4 do support the statement in [14] 
that model 2 should be applied for the modelling of moderately-concentrated ferrofluids, whereas model 3 should be taken for the modelling of stronglyconcentrated ferrofluids.



a)

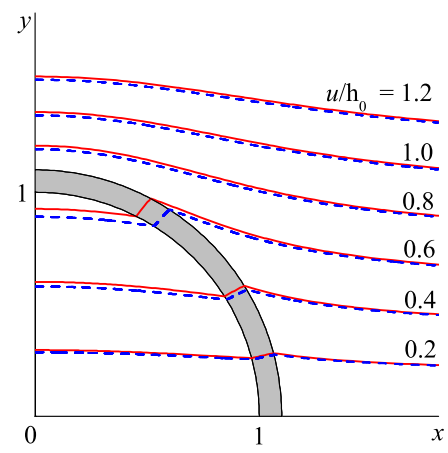

b)

Figure 5. Isolines of the dimensionless magnetostatic potential $u / h_{0}$ for the dimensionless layer thickness $\delta=1.1$, the Langevin susceptibility $\chi_{L}=7.61$, the dimensionless applied magnetic-field intensity $h_{0}=1$ (a) and $h_{0}=3$ (b). Dashed isolines correspond to model 2. Solid isolines correspond to model 3.

Figure 5 illustrates magnetic field structure in the computational domain by means of isolines of the magnetostatic potential. The isolines for model 2 and model 3 are close to each other for $\chi_{L}=7.61$. For any value of $\chi_{L}$ and $h_{0} \leq 1$, the magnetic field in the inner domain $\Omega_{1}$ is nearly uniform and vertically directed, as shown in Figure 5a. As expected, the inner magnetic field has lower intensity than the externally applied field $\left(h_{1}<h_{0}\right)$.

The computations, discussed in the paper, are made under assumption of a uniform ferroparticle distribution inside the ferrofluid. We have made test computations for the magnetostatics problem (model 1) coupled with the diffusion problem for ferromagnetic particles inside the ferrofluid in the case of a linear dependence of the magnetisation law on the concentration, when the diffusion problem is analytically solvable, see [25]. The numerical results for the coupled magnetostatics and diffusion problems, compared with the results of only model 1 for $\chi_{L} \leq 1$, are very close to each other.

\section{Conclusions}

Shielding properties of the cylindrical thick-walled ferrofluid layer, protecting against uniform magnetic fields, have been numerically investigated for the weakly-, moderately- and highly-concentrated ferrofluids. Computational procedure for the magnetic field, penetrating through the ferrofluid layer to the inner nonmagnetic domain, is developed. The computational algorithm is based on a coupled method of finite differences and boundary elements. The shielding effectiveness factor, estimating the decay factor of the externally applied magnetic field into the inner domain, is calculated for different ferrofluid properties, different layer thicknesses and different intensities of the applied 
field. Numerical results demonstrate that in weak fields $\left(H_{0}<10^{2} \mathrm{~A} / \mathrm{m}\right)$ the shielding effectiveness factor practically preserves a constant value, which depends on the initial susceptibility $\chi$ of the ferrofluid. Namely, $K_{\mathrm{ef}} \approx 1.4$ for the moderately-concentrated ferrofluid with $\chi=10$ and $K_{\mathrm{ef}} \approx 3.1$ for the dense ferrofluid with $\chi=50$. The shielding effect is almost absent in strong fields $\left(H_{0}>10^{6} \mathrm{~A} / \mathrm{m}\right)$. With respect to models 1,2 and 3 , one can conclude that the magnetodipole interparticle interactions in ferrofluids have significant influence to the shielding effectiveness factor and the mathematical formulation should depend on the ferrofluid under study. Namely, model 1 could be applied for the mathematical modelling of only weakly-concentrated ferrofluids, model 2 suits for moderately-concentrated ferrofluids, whereas model 3 should be used for the problem under study with application of strongly-concentrated ferrofluids.

\section{Acknowledgements}

The authors would like to thank Prof. G. Vainikko for useful comments concerning numerical solution of the system of integral Fredholm first-kind equations in the BEM algorithm. The authors are grateful to the reviewers for the valuable comments and helpful suggestions. In particular, we thank for the references to the publications, where analytical solutions for the shielding effectiveness factor in weak magnetic fields are discussed. We appreciate the suggestion of the reviewer to compare models 1-3 for the same values of the Langevin susceptibility. The authors are grateful to the Belarusian State Research Program "Convergence-2020" for the financial support of the project 1.5.01.3.

\section{References}

[1] H. Altenbach and G.I. Mikhasev(Eds.). Shell and Membrane Theories in Mechanics and Biology: From Macro- to Nanoscale Structures. Advanced structured materials. Springer, 2015. https://doi.org/10.1007/978-3-319-02535-3.

[2] J.-P. Berenger. Three-dimensional perfectly matched layer for the absorption of electromagnetic waves. J. of Computational Physics, 127(2):363-379, 1996. https://doi.org/10.1006/jcph.1996.0181.

[3] B.M. Berkovsky and V. Bashtovoi. Magnetic fluids and applications handbook. Begell House Inc. Publ., New York, 1996.

[4] B.M. Berkovsky, V.F Medvedev and M.S. Krakov. Magnetic fluids: engineering applications. Oxford University Press, Oxford, 1993.

[5] C.A. Brebbia, J.C.F. Telles and L.C. Wrobel. Boundary element techniques: theory and application in engineering. Springer-Verlag, Berlin, 1984. https://doi.org/10.1007/978-3-642-48860-3.

[6] A. Cebers, E. Blum and M.M. Maiorov. Magnetic fluids. Walter de Gruyter, Berlin, 1997.

[7] S. Celozzi, R. Araneo and G. Lovat. Electromagnetic Shielding. John Wiley \& Sons, 2008. https://doi.org/10.1002/9780470268483.

[8] Ya.G. Dorfman. Magnetic properties and structure of matter. Izdatelstvo LKI, Moscow, 2010. (in Russian) 
[9] E.A. Elfimova. Statistical thermodynamics and physical properties of magnetic fluids: influence of interparticle correlations. Dissertation for a degree of doctor, Ekaterinburg, 2016. (in Russian)

[10] V.T. Erofeenko, G.F. Gromyko and G.M. Zayats. Boundary value problems with integral boundary conditions for the modelling of magnetic fields in cylindrical film shells. Differential Equations, 53(7):935-948, 2017. https://doi.org/10.1134/S0012266117070102.

[11] U.V. Glonyagin. Elements of the theory and calculation of magnetostatic fields of ferromagnetic bodies. Sudostroenie, Leningrad, 1967. (in Russian)

[12] S.S. Grabchikov, A.V. Trukhanov, S.V. Trukhanov, I.S. Kazakevich, A.A. Solobay, V.T. Erofeenko and N.V. Vasilenkov. Effectiveness of the magnetostatic shielding by the cylindrical shells. J. Magn. Magn. Mater., 398(15):49-53, 2016. https://doi.org/10.1016/j.jmmm.2015.08.122.

[13] G.F. Gromyko, S.S. Grabchikov, V.T. Erofeenko and G.M. Zayats. The shielding effectiveness of static magnetic fields by cylindrical screen taking into account nonlinear effects. Physical Bases of Instrumentation, 4(4):30-39, 2015.

[14] A.O. Ivanov and O.B. Kuznetsova. Magnetic properties of dense ferrofluids: An influence of interparticle correlations. Physical Review E, 64:041405, 2001. https://doi.org/10.1103/PhysRevE.64.041405.

[15] L.V. King. Electromagnetic shielding at radio frequencies. Phil. Mag. J. Sci., 15(97):201-223, 1933. https://doi.org/10.1080/14786443309462178.

[16] O. Lavrova, G. Matthies, T. Mitkova, V. Polevikov and L. Tobiska. Numerical treatment of free surface problems in ferrohydrodynamics. Journal of Physics: Condensed Matter, 18(38):S2657-S2669, 2006. https://doi.org/10.1088/09538984/18/38/S09.

[17] O. Lavrova, V. Polevikov and L. Tobiska. Instability of a magnetic fluid drop in a capillary: a numerical study. Magnetohydrodynamics, 44(2):183-189, 2008.

[18] O. Lavrova, V. Polevikov and L. Tobiska. Numerical study of the Rosensweig instability in a magnetic fluid subject to diffusion of magnetic particles. Math. Model. Anal., 15(2):223-233, 2010. https://doi.org/10.3846/13926292.2010.15.223-233.

[19] O. Lavrova, V. Polevikov and L. Tobiska. Modeling and simulation of magnetic particles diffusion in a ferrofluid layer. Magnetohydrodynamics, 52(4):417-430, 2016.

[20] A.V. Lebedev. Dipole interparticle interaction in magnetic fluids. Colloid Journal, 76(3):334-341, 2014. https://doi.org/0.1134/S1061933X14030107.

[21] G. Mikhasev, I. Mlechka and H. Altenbach. Soft suppression of traveling localized vibrations in medium-length thin sandwich-like cylindrical shells containing magnetorheological layers via nonstationary magnetic field. In J. Awrejcewicz(Ed.), Dynamical Systems: Theoretical and Experimental Analysis, volume 182 of Springer Proceedings in Mathematics \& Statistics, pp. 241-260, Switzerland, 2016. Springer.

[22] G.I. Mikhasev, H. Altenbach and E.A. Korchevskaya. On the influence of the magnetic field on the eigenmodes of thin laminated cylindrical shells containing magnetorheological elastomer. Composite Structures, 113:186-196, 2014. https://doi.org/10.1016/j.compstruct.2014.02.031. 
[23] V.K. Polevikov. Methods for numerical modeling of two-dimensional capillary surfaces. Computational Methods in Applied Mathematics, 4(1):66-93, 2004. https://doi.org/10.2478/cmam-2004-0005.

[24] V.K. Polevikov and B.T. Erofeenko. Numerical modelling of the interaction of a magnetic field with a cylindrical magnetic-fluid layer. Informatika, 2(54):5-13, 2017. (in Russian)

[25] V.K. Polevikov and L. Tobiska. On the solution of the steady-state diffusion problem for ferromagnetic particles in a magnetic field. Math. Model. Anal., 13(2):233-240, 2008. https://doi.org/10.3846/1392-6292.2008.13.233-240.

[26] A.F. Pshenichnikov and A.V. Lebedev. Low-temperature susceptibility of concentrated magnetic fluids. J. Chem. Phys., 121(11):5455-5467, 2004. https://doi.org/10.1063/1.1778135.

[27] A.F. Pshenichnikov and A.V. Lebedev. Magnetic susceptibility of concentrated ferrocolloids. Colloid Journal, 67(2):189-200, 2005. https://doi.org/10.1007/s10595-005-0080-x.

[28] R.E. Rosensweig. Ferrohydrodynamics. Dover Pubns, New York, 1998.

[29] A.N. Tikhonov and A.A. Samarskii. Equations of Mathematical Physics. Pergamon, Oxford, 1963.

[30] D.I. Tishkevich, S.S. Grabchikov, S.B. Lastovskii, S.V. Trukhanov, T.I. Zubar, D.S. Vasin and A.V. Trukhanov. Correlation of the synthesis conditions and microstructure for Bi-based electron shields production. J. Alloys Compd., 749:1036-1042, 2018. https://doi.org/10.1016/j.jallcom.2018.03.288.

[31] D.I. Tishkevich, S.S. Grabchikov, L.S. Tsybulskaya, V.S. Shendyukov, S.S. Perevoznikov, S.V. Trukhanov, E.L. Trukhanova, A.V. Trukhanov and D.A. Vinnik. Electrochemical deposition regimes and critical influence of organic additives on the structure of Bi films. J. Alloys Compd., 735:1943-1948, 2018. https://doi.org/10.1016/j.jallcom.2017.11.329.

[32] A.V. Trukhanov, S.S. Grabchikov, A.A. Solobai, D.I. Tishkevich, S.V. Trukhanov and E.L. Trukhanova. AC and DC-shielding properties for the Ni80Fe20/Cu film structures. J. Magn. Magn. Mater., 443:142-148, 2017. https://doi.org/10.1016/j.jmmm.2017.07.053.

[33] T.I. Zubar, L.V. Panina, N.N. Kovaleva, S.A. Sharko, D.I. Tishkevich, D.A. Vinnik, S.A. Gudkova, E.L. Trukhanova, E.A. Trofimov, S.A. Chizhik, S.V. Trukhanov and A.V. Trukhanov. Anomalies in growth of electrodeposited Ni-Fe nanogranular films. CrystEngComm, 20:2306-2315, 2018. https://doi.org/10.1039/C8CE00310F.

[34] T.I. Zubar, S.A. Sharko, D.I. Tishkevich, N.N. Kovaleva, D.A. Vinnik, S.A. Gudkova, E.L. Trukhanova, E.A. Trofimov, S.A. Chizhik, L.V. Panina, S.V. Trukhanov and A.V. Trukhanov. Anomalies in NiFe nanogranular films growth. J. Alloys Compd., 748:970-978, 2018. https://doi.org/10.1016/j.jallcom.2018.03.245. 